\title{
Plasma surface interactions in impurity seeded plasmas
}

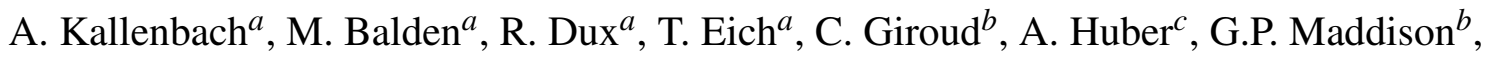

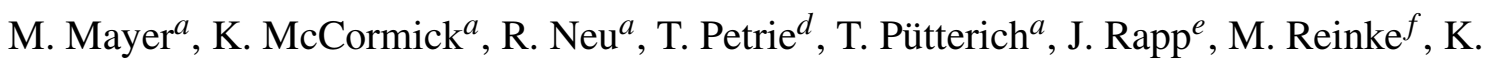

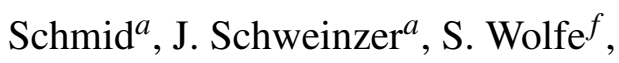
and the ASDEX Upgrade Team, DIII-D Team, Alcator Team, JET-EFDA Contributors ${ }^{g,+}$ ${ }^{a}$ Max-Planck-Institut für Plasmaphysik, EURATOM Association, Garching, Germany ${ }^{b}$ EURATOM/CCFE Fusion Association, Culham Science Centre, Abingdon, OX14 3DB, $U K$

${ }^{c}$ FZ Jülich GmbH, Institut f. Plasmaphysik, Association EURATOM-FZJ, Jülich, Germany ${ }^{d}$ General Atomics, San Diego, California, USA

${ }^{e}$ FOM Institut voor Plasma Fysica Rijnhuizen, EURATOM Association, TEC, Nieuwegein, The Netherlands

${ }^{f}$ Massachusetts Institute of Technology, Plasma Science and Fusion Center, Cambridge, USA

${ }^{g}$ JET-EFDA, Culham Science Centre, Abingdon, OX14 3DB, UK

\begin{abstract}
With tokamak devices developing towards higher heating powers, and carbon plasma facing components being increasingly replaced by high-Z materials like tungsten, impurity seeding for radiative power dissipation gains more importance. This review summarizes the core and divertor radiative characteristics of potential seeding species, namely noble gases and nitrogen. Due to its radiative capability below $10 \mathrm{eV}$, nitrogen turns out to be a suitable replacement for carbon as a divertor radiator. For typical plasma parameters and high radiation levels, it becomes the most important eroding species for high-Z plasma fac-
\end{abstract}


ing components. Nitrogen exhibits pronounced storage in near surface tungsten layers in an about 1:1 W/N atomic ratio, which may effect $\mathrm{W}$ sputtering. While the inter-ELM erosion of tungsten can be almost completely eliminated by electron temperature reduction, type-I ELMs remain an effective sputtering source. Since a large ELM cannot be significantly ameliorated by radiation, impurity seeding has to be integrated with a benign ELM scenario, like the type-III ELMy H-mode or active ELM control by pellets or resonant magnetic perurbations.

Key words:

PACS: 52.55.Fa, 52.55.Rk, 52.25.Vy, 52.40.Hf

JNM keyword codes: P0500, P0600, D0500, T1000

PSI-19 keywords: Radiation, Divertor, Impurity, Sputtering

${ }^{*}$ Corresponding author address: Boltzmannstr. 2, D-85748 Garching, Germany

*Corresponding author email: Arne.Kallenbach@ipp.mpg.de

${ }^{*}$ Corresponding author phone: +49-89-3299 1721

${ }^{*}$ Corresponding author fax: +49-89-3299 1812 


\section{Introduction}

Impurity seeding has been used for a long time in tokamak research [1]. Originally, a major focus was the improvement of energy confinement, which was often observed in radiative regimes in combination with electron density profile peaking [2], [3], [4]. However, this topic calmed down after these improved confinement regimes could not be fully reproduced in larger devices under low collisionality conditions [5] [6] [7]. The reduction of limiter and divertor power loads was also an early experimental goal in view of future devices [8], but not an experimental necessity [9] [10] . This changed the first time for ASDEX Upgrade (AUG), where the intrinsic impurity level decreased considerably after completion of the coating of plasma facing components (PFCs) with tungsten (W) and wall conditioning by boronization [11]. Feedback controlled radiative cooling became mandatory for high heating power scenarios [12]. The same is foreseen for the ITER like wall (ILW) in JET, where beryllium walls and a tungsten divertor are expected to lead to reduced intrinsic radiative levels [13]. ITER itself will heavily rely on impurity seeding, at least during its nuclear phase with high power discharges and a tungsten divertor. A future reactor will have to radiate several hundreds of megawatts [14] [15], and power exhaust is one of the major design issues.

The experimental task is more difficult than just puffing radiating species into a discharge, since this has to be embedded into a tolerable edge localized mode (ELM) scenario. The presence of seed impurities does not only reduce the plasma temperature in front of material surfaces. It also adds a new plasma species which leads to surface material sputtering.

$\overline{1+}$ See the Appendix of F. Romanelli et al., Proceedings of the 22nd IAEA Fusion Energy Conference 2008, Geneva, Switzerland 
Depending on the seeded species and surface material, modifications of the surface structure have been observed. Only noble gases and nitrogen are considered as seed impurities, since non-recycling impurities such as carbon would lead to tritium co-deposition. Tungsten is considered as the wall material, since plasma-wall interaction with seeding species is in particular an issue for high-Z elements.

This paper is organized as follows. In section 2, basic data for seed impurity radiation under divertor conditions and $\mathrm{W}$ and Mo sputtering yields are summarized. The integration of radiative cooling into a small ELM scenario is addressed in section 3. Since nitrogen is a promising candidate to produce partial divertor detachment without pronounced degradation of the energy confinement, some issues of nitrogen-tungsten plasma-wall interaction investigated in laboratory experiments are discussed in section 4 . Measured $\mathrm{N} \rightarrow \mathrm{W}$ sputtering yields between and during ELMs are shown in section 5. Finally, some conclusions for required future work in the field are drawn.

\section{Basic effects during impurity seeding}

\subsection{Core and divertor radiative losses}

Radiative power removal, with the ultimate goal of achieving partial detachment of the outer divertor, can be regarded as a 3-stage process [8]. First, main plasma radiation is used to reduce the power flux over the separatrix. The radiative loss function $\mathrm{L}_{z}$ of an impurity ion, which can be calculated for core plasma conditions assuming coronal equilibrium, scales roughly with the atomic charge $\mathrm{Z}^{3}$ (see figure 1c). Therefore, heavier species are the more efficient seed species for core radiation, since they cause less fuel dilution. Core radiative losses have generally to be limited to avoid negative effects on energy confinement, or a too 
close approach of the H-L transition power threshold. In first approximation, core radiative losses are expected to scale linearly with the impurity concentrations.

The second step in radiative power removal is scrape-off layer (SOL) and divertor impurity radiation. Here a relatively low- $Z$ species is best suited, which has a favourable radiative characteristics in the $10 \mathrm{eV}$ temperature range, while it fully ionizes under core conditions (dilution is not considered here). Since the impurity residence time in the divertor may be shorter than ionisation and recombination time scales, the radiative losses cannot be calculated assuming coronal equilibrium. For these conditions, the ansatz of a radiative potential [17] is more appropriate: A recycled or puffed neutral impurity atom enters the divertor plasma. While being subsequently ionised to higher charge states, line radiation is emitted. The radiated energy per atom can be calculated using atomic data and a collisonal-radiative model (here, the ADAS database is used [16]). It is useful to specify a particle residence time in the divertor plasma after which the evolution of the collisional-radiative processes is terminated [8]. Figures 1a,b show the corresponding radiative loss function for species of interest under divertor conditions for two different values of the product of the density and the residence time. The radiative losses for the conditions of figures $1 \mathrm{a}, \mathrm{b}$ correspond to the particle influx picture used in the spectroscopic S/XB method [18]: Photons are emitted from neutrals or ions in low ionization stages during the time required for ionization to the next stage. Since both the ionisation time and the excitation time depend about linearly on the electron density (as long as multi-step processes are not important), the radiated power density per impurity atom depends only weakly on the electron density for conditions where recombination is not important. For long residence times and higher emitting ionization stages, the radiative losses decrease towards their coronal values - the non-coronal conditions in a divertor plasma increase the radiative losses. The divertor radiation does not generally increase linearly with 
the impurity concentration. This is caused by the reduction of the parallel conductivity due to the rise in effective charge $\mathrm{Z}_{e f f}$, which leads to a steepening of the parallel electron temperature gradient and which can therefore lead to a shrinking of the strongly radiating zone [19].

The final step towards acceptable peak power loads on a divertor target is the achievement of partial, i.e. strike point detachment. This relies on momentum losses leading to a pressure drop in front of the target. The most important momentum loss terms are recombination (radiative and three-body) and charge exchange of hot ions with cold neutrals. A relatively cold plasma is required to make these momentum loss mechanisms effective [20]. Due to the strongly negative electron temperature dependence of recombination rate coefficients, this process takes effect only below about $3 \mathrm{eV}$, the recombination rate equals the ionization rate around about $1 \mathrm{eV}$. Charge exchange needs high neutral fluxes, which also occur under cold, high recycling conditions. Besides direct power dissipation, a major task of seed impurity radiation is to cool the plasma down to temperatures where momentum losses start to become effective. It should be noted that momentum loss processes and the detachment observed in tokamaks are currently not fully reproduced by edge modelling [21].

Inspecting figure 1 reveals that for $\mathrm{T}_{e}$ below $10 \mathrm{eV}$, nitrogen and argon come closest to carbon, which is known as effective intrinsic coolant in devices with carbon plasma facing components [22] [23] [24]. Neon exhibits a comparatively low cooling capacity at low electron temperatures. Argon has to be ruled out as strong divertor radiator in devices with moderate divertor impurity compression and low pumping speeds, since the required divertor concentrations in the per cent range are not compatible to the maximum allowed core concentration of a few per mille. An exception is the DIII-D puff-and-pump scenario [25]. Here, strong pumping achieved by placing the strike point at the entrance of the pump throat allowed to 
overcome the divertor-main plasma argon exchange rate. For devices without such strong pumping (AUG, JET, ITER), a mix of at least 2 seeding species should be used to optimize the spatial radiation distribution [10] [26], with $\mathrm{N}$ being the best option for the divertor.

\subsection{Sputtering of the plasma facing material}

Due to the high energy threshold of the deuterium sputtering yield (see figure 2), sputtering of high-Z target materials occurs mainly by impurity ions [27]. High energy particles expelled from the core by ELMs or other MHD instabilities may contribute to the sputtering rate. Since the high-Z PFC material has to remain below a concentration limit in the plasma center, care must be taken that the seeded species does not lead to too high sputtered fluxes. Figure 2 shows effective sputtering yields for seed impurity candidates for $\mathrm{W}$ and Mo. Shown are effective yields, normalized to the hydrogenic flux, assuming typical impurity concentrations in the impinging flux [28].

For the typical seed impurity concentrations assumed in the plot, quite similar W sputtering rates are obtained for different impurities in the low electron temperature region. Overall, the low-Z divertor radiating species appears to be the more important sputtering source compared to potential core radiators. Sputtering yields for Mo are shifted towards lower temperatures and slightly higher values due to the lower mass of Mo compared to W. Obviously, the absolute temperature (or ion impact energy) becomes very important when very low sputtered fluxes are aspired. The sputtering yield of $\mathrm{W}$ by nitrogen has been obtained from a TRIM code calculation neglecting effects of nitrogen storage in near surface layers, which may lead to a reduction of the sputtering yield [30]. 


\subsection{Energy confinement issues}

A thorough discussion of energy confinement behaviour with impurity seeding is beyond the scope of this review. Nevertheless, a few remarks are required since sufficient confinement is a necessary boundary condition for each radiative scenario. Originally, in small-medium size devices and at quite high collisionalities, impurity seeding often caused electron density peaking and improved energy confinement [2], [3]. This behaviour has been attributed to the reduction of ion temperature gradient mode (ITG) turbulence caused by a rise in the effective ion charge in combination with steepening of the density gradients [31]. In larger devices, both confinement improvement and density profile peaking appeared much more reluctant. In JET as well as in different JT-60U long pulse scenarios, the radiative losses caused mostly a reduction of energy confinement [32], [33], [10]. Again a different behaviour showed up under high power conditions in AUG, where energy confinement increased by up to $30 \%$ with strong nitrogen seeding [34]. The improvement is caused by increased temperatures in the core and at the pedestal, while no changes in the density profile shape occur. A related behaviour may be the partial recovery of the confinement reduction associated with the typeIII pedestal observed in a nitrogen seeded JET hybrid scenario, where core temperatures slightly increased with increasing radiative fraction [35]. Quantitative theoretical predictions of the effect of impurity seeding on core plasma profiles are difficult, in particular in the transitional region between dominant ITG and trapped electron mode (TEM) instabilities, which have opposite trends of the collisionality dependence of the density gradient [36] [37]. In the pedestal region, significant fuel dilution by nitrogen may increase the stability via its influence on the ion pressure gradient. Core dilution has also been found to reduce the ITG growth rates in gyrokinetic calculation with the GS2 code for AUG conditions [38]. 


\subsection{Global experimental observations}

A pronounced reduction of the inter-ELM divertor heat load by impurity seeding has been clearly observed on several devices. Figure 3 shows power load profiles from thermography with $\mathrm{N}$ and $\mathrm{Ne}$ seeding [33] compared to the unseeded case with the same D puff level in JET. An unpuffed case is also shown to demonstrate substantial heat load. Clearly for JET, a price in terms of confinement reduction has to be paid when the heat loads are ameliorated by impurity seeding. It should be noted that the $\mathrm{N}$ puff rate to obtain moderate power loads is a factor of 10 higher than the corresponding Ne puff rate. This factor is larger than what would be expected from the atomic radiation data, and is explained by the retention of nitrogen in plasma facing components (see Section 4). In early Alcator C-Mod experiments, divertor radiation and detachment were achieved with nitrogen seeding, but not with neon and argon [9]. Recent seeding experiments in enhanced $\mathrm{D}_{\alpha}$ (EDA) H-modes showed comparable performance of $\mathrm{N}$ and $\mathrm{Ne}$, both performing better than $\mathrm{Ar}$ [39]. In the fully tungsten coated AUG, increased divertor radiation has been inferred from bolometry tomographic reconstruction during $\mathrm{N}$ seeding, showing a similar radiation pattern compared to previous carbon cases [34].

Summarizing the experience from AUG, earlier Alcator C-Mod experiments and JET (albeit with less clear evidence), nitrogen appears to be an attractive seeding species for divertor cooling, in agreement with the expectations arising from the atomic data. If core radiative losses are to be enhanced, higher $\mathrm{Z}$ species should be added. A mixture of nitrogen with a heavier noble gas should give optimum results, where the higher- $Z$ species are expected to play a minor role for PWI issues due to their lower wall fluxes. 


\section{Impurity seeding and small ELM scenarios}

Due to limitations of divertor target materials, only very small ELMs will be acceptable for ITER. Radiative buffering of ELMs by intrinsic and seed impurities is only effective for very small ELMs below about $20 \mathrm{~kJ}$ for JET conditions. This has been demonstrated in JET experiments as well as with Edge2d/NIMBUS edge modelling [40], and is shown in figure 4. Consequently, if low target energies are required, ELMs have to start at the pedestal already as small ELMs. The radiative seeding scenario, which acts predominantly on the inter-ELM power load, needs to be integrated with ELM mitigation. A small ELM scenario which is compatible with impurity seeding and possibly suffices with ITER heat load limits is the type-III ELMy H-mode [41]. Since it generally exhibits reduced confinement, it may require ITER operation at slightly increased current of $17 \mathrm{MA}$ to achieve $\mathrm{Q}=10$. Small ELMs have also been obtained in ASDEX Upgrade high power discharges combining strong D and $\mathrm{N}$ puff levels. In the vicinity of the type-III - type-I ELM transition, an energy confinement measure $\mathrm{H}_{98 y, 2}=1$ has been obtained from both ELM types at small ELM sizes [42]. Type-II, grassy or EDA ELM regimes also exhibit small ELM energies, but their feasibility in ITER is questionable. Active ELM control is the most preferred option, if applicable to ITER. It has also to be shown that these techniques comply with impurity seeding, which is necessary to ameliorate the inter-ELM target heat load. No special problems regarding impurity seeding are expected for mitigation techniques which reduce the ELM size by increasing the ELM frequency, like pellet pacemaking, or vertical kicks. First compatibility studies with resonant magnetic perturbation (RMP) ELM suppression have recently been performed on DIII-D [43]. Figure 5 shows the peak heat flux in the inner divertor versus the radiative fraction. Substantial peak heat fluxes during type-I ELMs are measured without RMP and gas injection (black squares). When the RMP coils are activated under prescribed conditions, ELMs 
are suppressed resulting in a low peak heat flux (green crosses). The ELMs reappear, as the edge density is raised during deuterium and argon gas puffing (red circles). The D puff is required to maintain a low core Ar content when Ar is injected for radiative divertor operation. The peak heat flux decreases with increasing radiative fraction. While ELM suppression so far could not be maintained at higher D puff rates, RMP operation reduces the ELM peak heat flux compared to pre-RMP cases with similar radiative fraction by about $25 \%$. Energy confinement is degraded by a similar amount, but does not decrease with increasing radiative fraction, $\mathrm{H}_{98(y, 2)}$ is about 0.9 at the highest $\mathrm{P}_{r a d} / \mathrm{P}_{i n}=0.8$. Since this result represents an initial study, significant gains can be expected for a future optimization of the combination of RMP operation, D puff and (mixed) impurity injection.

\section{Surface material modifications due to seed impurities}

Divertor and limiter surfaces experience high fluxes of impinging impurity ions. These inevitably leads to sputtering, but may also cause more sophisticated changes in the near surface structure. In particular nitrogen exhibits subtle effects like strong wall latency or even chemical reactions with carbon [45]. Considering possible surface modifications by the impurity species, we first look at the penetration depth into tungsten for typical edge plasma conditions as shown in figure 6a, where diffusion of the implanted species has been neglected. The penetration scales approximately with the velocity of the seed species, and is about $1 \mathrm{~nm}$ for nitrogen divertor conditons. Nitrogen is stored in tungsten very effectively via the formation of nitride states. Laboratory measurements find saturation of nitrogen storage at an atomic ratio of about $1: 1 \mathrm{~N}: \mathrm{W}$ over the penetration depth for room temperature conditions. Implantations performed at higher temperatures indicated that $\mathrm{WN}$ becomes unstable above $\approx 600 \mathrm{~K}[30]$. The formation of the nitride state is the reason for the much stronger 
$\mathrm{N}$ wall storage compared to noble gases. Figure $6 \mathrm{~b}$ shows measured and calculated sputter yields for tungsten under nitrogen bombardment. Measurements were taken on thin $(\approx 500$ nm) tungsten layers on silicon substrates. A reduced sputter yield is predicted by the dynamic TRIM calculations as observed in the laboratory experiment [30]. A reduction of the yield compared to the static TRIM calculation, which does not take the effect of $\mathrm{N}$ storage into account, is clearly visible. The reduction can be understood as the effect of collisions of the impacting $\mathrm{N}$ with $\mathrm{N}$ in the material, which reduces the sputtering of $\mathrm{W}$. Possible changes of the microscopic stability of the nitridic material have not been taken into account.

Comparison of the laboratory results with tokamak conditions revealed a qualitatively similar storage behavior. However, an order of magnitude higher nitrogen storage areal density was found in AUG [12]. Since a different penetration is not expected, either a surface structure or effects related to deposited (B, C) impurity layers are supposed to be responsible for the different behaviour.

Figure 7 shows a scanning electron micrograph (SEM) picture of an ASDEX Upgrade outer divertor tile. A very rough surface structure can be seen, which is due to the crystalline growth of the $\mathrm{W}$ layer during the manufacturing process. This roughness is much larger compared to the $\mathrm{N}$ penetration depth of about $1 \mathrm{~nm}$. The enhanced surface area caused by the roughness at least partially explains the enhanced $\mathrm{N}$ storage capacity in the tokamak. However, the small-scale (few $100 \mathrm{~nm}$ ) roughness due to the crystalic structure of the $\mathrm{W}$ layers has vanished after an experimental campaign due to the combined effect of erosion/redeposition processes. Only a larger-scale roughness (of the order of a few $\mu \mathrm{m}$ ) at least partly caused by the surface structure of the carbon substrate has survived. The re-deposited W layers were found to contain co-deposited $\mathrm{B}, \mathrm{C}$ and $\mathrm{N}$. This co-deposition with $\mathrm{N}$ may form another possibility for $\mathrm{N}$ storage. 


\section{Tungsten sputtering by nitrogen under tokamak conditions}

The pronounced differences in $\mathrm{N}$ storage behaviour between laboratory and tokamak conditions discussed in the preceding section suggest a benchmark of the sputtering behaviour under tokamak conditions. We compare two AUG discharges with low and high nitrogen fueling level, feedback-controlled on inter-ELM divertor temperature [12]. Both discharges have $11 \mathrm{MW}$ heating power and a Greenwald density fraction of 0.6. The discharge with the higher nitrogen seeding rate has an about a factor 2 higher ELM frequency, and a factor 3 shorter ELMs, showing pronounced ELM mitigation by nitrogen injection. Average ELM energies and $\mathrm{H}$-factors are $\mathrm{W}_{E L M}=60 \mathrm{~kJ}$ and $\mathrm{H}_{98, y 2}=0.95$ with low $\mathrm{N}$ level and about $\mathrm{W}_{E L M}=20 \mathrm{~kJ}$ and $\mathrm{H}_{98, y 2}=1.1$ with the higher level. Figure 8 shows the neutral tungsten influx close to the spatial peak flux location a few $\mathrm{cm}$ in the SOL direction of the strike point. Measurements are taken with $0.2 \mathrm{~ms}$ temporal resolution [27], coherent ELM averaging has been performed over $0.6 \mathrm{~s}$ discharge time to improve the statistics. Obviously, the measurement of the inter-ELM W flux is a challenge, taking into account the very large ELM peaks and the weak inter-ELM signal. Negative individual data points are caused by detector noise and the subtraction of background spectra without plasma. On the positive side, with nitrogen almost complete suppression of inter-ELM W sputtering in the divertor is possible. Time dependent divertor parameters, measured by a Langmuir probe very close to the WI emission location, are shown in figure 9. The combination of the data shown in figures 8 and 9 allows to calculate the sputtering yield assuming $\mathrm{T}_{i}=\mathrm{T}_{e}$ if the nitrogen fraction in the impinging ion flux is known. We take the corresponding main plasma $\mathrm{N}$ concentrations, derived from $\mathrm{Z}_{\text {eff }}$ measurements, multiplied by the divertor enrichment factor, which is assumed as 2 . The resulting $\mathrm{N}$ fraction in the ion flux is 1 and 4 per cent for the low and high $\mathrm{N}$ cases, respectively. Obviously, the procedure described above bears significant uncertainties. The evaluation of the 
Langmuir probes is more uncertain during ELMs, where non-thermal electrons may influence the derived electron temperature. During ELMs, deuterium ions lost from the pedestal may increase the sputtered tungsten flux. Further sources of uncertainty are the very transient signals during an ELM and the marginal neutral tungsten line (WI) signal in between ELMs, as shown in figure 8 . The sputtering yields derived under the described assumptions shown in figure 10 are compatible to the theoretical curves. The large errror bars do not allow to conclude on possible variations of the sputter yield due to the presence of $\mathrm{N}$ in $\mathrm{W}$. The tungsten divertor source is dominated by the ELM contribution under these conditions. It is not easy to assess the influence of the ELM W divertor source on the plasma tungsten content. Due to the higher divertor temperature and density during an ELM, the ionisation length of sputtered neutral tungsten decreases and the promptly redeposited fraction increases [48]. Until these effects have been included into current 2D codes, only qualitative comparisons with measurements are possible. Regarding the sputtering yields inferred from tokamak experiments, the large uncertainties involved do not allow to decide on a modification of the erosion yield by the nitrogen stored in the near-surface tungsten layer.

\section{Conclusions}

Impurity seeding in future high power devices, with plasma facing components probably covered with tungsten or a tungsten alloy will require a well adapted and optimized scenario. Ar or $\mathrm{Kr}$ are suitable core and pedestal radiators, with concentrations in the per mille range. In ASDEX Upgrade, nitrogen seeding enhances the divertor radiation, while $\mathrm{Ne}$ and Ar radiate predominantly in a radiative mantle. This behaviour is compatible with the atomic data presented in figure 1 . The core/mantle radiation causes a reduced power flux into the divertor, which typically leads to a net decrease of divertor radiation. The situation is less clear in 
C-Mod and JET. In JET, a reduction of energy and particle confinement by strong seeding may even lead to a reduction of radiation caused by a reduced electron density. In DIII-D, an increase of the divertor radiation with Ar could be achieved in scenarios with high divertor compression. Detailed bolometry tomographic deconvolutions and systematic inter-machine comparisons are needed to obtain a universal picture and a more robust extrapolation to future devices. More work is also required to disentangle the effect of impurity seeding on energy confinement. Different effects of the seeding have been observed on pedestal temperature and changes of the ELM frequency and size.

In case that high divertor seed radiation levels are generated with nitrogen, $\mathrm{N}$ concentrations of several per cent occur in the divertor plasma, therefore nitrogen will be the dominant sputtering species. The plasma core will be mainly affected by dilution and bremsstrahlung. Moreover, the strong uptake and storage of nitrogen in the near surface tungsten layers could even change its material properties, as it has been observed as blister formation in mixed DHe bombardment of tungsten [49]. Extension of seeding scenarios to higher heating powers, aiming towards the ITER P/R (power divided by major radius) value of $20 \mathrm{MW} / \mathrm{m}$, will help to identify limitations of the technique and highlight plasma-wall interaction issues. Impurity seeding needs to be integrated into a small or suppressed ELM scenario. No particular problems related to impurities are expected for methods relying on increasing the ELM frequency. Combination with RMP operation has just started, and first positive results with mitigated ELMs have been obtained on DIII-D. Naturally mitigated ELMs are observed on ASDEX Upgrade with strong nitrogen puffing, but portability of this effect to larger machines is open.

\section{Acknowledgement}

This work was supported by EURATOM and carried out within the framework of the Eu- 
ropean Fusion Development Agreement. The views and opinions expressed herein do not necessarily reflect those of the European Commission.

\section{References}

[1] E. A. Lazarus, J. D. Bell, C. E. Bush, et al., Nucl. Fusion 25 (1985) 135.

[2] A. M. Messiaen, J. Ongena, U. Samm, et al., Nucl. Fusion 34 (1994) 825.

[3] O. Gruber, A. Kallenbach, M. Kaufmann, K. Lackner, V. Mertens, et al., Phys. Rev. Lett. 74 (1995) 4217.

[4] A. Kallenbach, R. Dux, V. Mertens, O. Gruber, G. Haas, et al., Nucl. Fusion 35 (1995) 1231.

[5] P. Dumortier, P. Andrew, G. Bonheure, R. V. Budny, R. Buttery, et al., Plasma Phys. Controlled Fusion 44 (2002) 1845.

[6] J. Ongena, P. Monier-Garbet, W. Suttrop, P. Andrew, M. Bécoulet, et al., Nucl. Fusion 44 (2004) 124.

[7] P. Monier-Garbet, P. Andrew, P. Belo, G. Bonheure, Y. Corre, et al., Nucl. Fusion 45 (2005) 1404.

[8] D. Post, J. Abdallah, R. E. H. Clark, and N. Putvinskaya, Phys. Plasmas 2 (1995) 2328.

[9] J. Goetz, B. LaBombard, B. Lipschultz, et al., Phys. Plasmas 6 (1999) 1899.

[10] N. Asakura, T. Nakano, T. Sakamoto, et al., Nucl. Fusion 49 (2009) 115010.

[11] A. Kallenbach, R. Dux, M. Mayer, R. Neu, T. Pütterich, et al., Nucl. Fusion 49 (2009) 045007.

[12] A. Kallenbach, R. Dux, J. Fuchs, et al., Plasma Phys. Controlled Fusion 52 (2010) 055002.

[13] G. Maddison, K. McCormick, C. Giroud, et al., in Europhysics Conference Abstracts (Proc. of the 36th EPS Conference on Controlled Fusion and Plasma Physics, Sofia, 2009), volume 33E, pages P-2.160, 2009. 
[14] G. Pacher, H. Pacher, G. Janeschitz, et al., Nucl. Fusion 47 (2007) 469.

[15] K. Tobita, S. Nishio, M. Enoeda, et al., Nucl. Fusion 49 (2009) 075029.

[16] H. P. Summers, Atomic data and analysis structure users manual, JET-IR 06 (Abingdon: JET Joint undertaking) (1994), http://open.adas.ac.uk/

[17] U. Samm, H. Bogen, P. an Claassen, et al., Journal of Nuclear Materials 176-177 (1990) 273.

[18] K. Behringer, H. P. Summers, B. Denne, M. Forrest, and M. Stamp, Plasma Phys. Controlled Fusion 31 (1989) 2059.

[19] K. Lackner and R. Schneider, Fusion Eng. and Design 22 (1993) 107.

[20] C. S. Pitcher, A. Herrmann, H. Murmann, H. Reimerdes, J. Schweinzer, et al., Plasma Phys. Controlled Fusion 39 (1997) 1129, preprint in IPP 1/295, Januar 1997.

[21] M. Wischmeier, M. Groth, A. Kallenbach, A. V. Chankin, D. P. Coster, et al., J. Nucl. Mater. 390-391 (2009) 250.

[22] M. Fenstermacher, S. Allen, N. Brooks, et al., Phys. Plasmas 4 (1997) 1761.

[23] A. Kallenbach, M. Kaufmann, D. P. Coster, J. C. Fuchs, A. Herrmann, et al., Nucl. Fusion 39 (1999) 901.

[24] T. Nakano, H. Kubo, N. Asakura, et al., J. Nucl. Mater. 390-391 (2009) 255.

[25] T. Petrie, N. Brooks, M. Fenstermacher, et al., Nucl. Fusion 48 (2008) 045010.

[26] R. Neu et al., P2-22, this conference, to be published in Journal of Nuclear Materials .

[27] R. Dux, V. Bobkov, A. Herrmann, A. Janzer, A. Kallenbach, et al., J. Nucl. Mater. 390-391 (2009) 858 .

[28] B. Unterberg, M. Brix, R. Jaspers, et al., J. Nucl. Mater. 266-269 (1999) 75.

[29] W. Eckstein et al., Sputtering data, Report IPP 9/82, IPP Garching, 1993. 
[30] K. Schmid, A. Manhard, C. Linsmeier, et al., Nucl. Fusion 50 (2010) 025006.

[31] M. Z. Tokar, R. Jaspers, H. R. Koslowski, et al., Plasma Phys. Controlled Fusion 41 (1999) B317.

[32] G. F. Matthews, B. Balet, S. J. Davies, et al., Nucl. Fusion 39 (1999) 19.

[33] G. Maddison et al., O-23, this conference, to be published in Journal of Nuclear Materials .

[34] O. Gruber, A. C. C. Sips, R. Dux, T. Eich, J. C. Fuchs, et al., Nucl. Fusion 49 (2009) 115014.

[35] Y. Corre, E. Joffrin, P. Monier-Garbet, Y. Andrew, G. Arnoux, et al., Plasma Phys. Controlled Fusion 50 (2008) 115012.

[36] C. Angioni, E. Fable, M. Greenwald, et al., Plasma Phys. Controlled Fusion 51 (2009) 124017.

[37] E. Fable, C. Angioni, and O. Sauter, Plasma Phys. Controlled Fusion 52 (2010) 015007.

[38] G. Tardini, R. Fischer, V. Igochine, et al., in Europhysics Conference Abstracts (Proc. of the 36th EPS Conference on Controlled Fusion and Plasma Physics, Sofia, 2009), volume 33E, pages O2.004, 2009 .

[39] M. Reinke et al., P2-25 this conference, to be published in Journal of Nuclear Materials .

[40] J. Rapp, P. Monier-Garbet, G. Matthews, et al., Nucl. Fusion 44 (2004) 312.

[41] J. Rapp, Y. Corre, Y. Andrew, et al., Nucl. Fusion 49 (2009) 095012.

[42] J. Rapp et al., to be published (2010).

[43] T. Petrie, T. Evans, M. Fenstermacher, et al., P1-44, this conference, to be published in Journal of Nuclear Materials .

[44] C. Ruset, E. Grigore, H. Maier, et al., Physica Scripta T128 (2007) 171.

[45] F. L. Tabarés, D. Tafalla, V. Rohde, M. Stamp, G. Matthews, et al., J. Nucl. Mater. 337-339 (2005) 867. 
[46] D. Nishijima, R. Doerner, M. Baldwin, et al., Phys. Plasmas 16 (2009) 122503.

[47] M. Wischmeier, A. Kallenbach, A. V. Chankin, D. P. Coster, T. Eich, et al., J. Nucl. Mater. 363-365 (2007) 448.

[48] T. Pütterich et al., I-14, this conference, to be published in Journal of Nuclear Materials .

[49] M. Miyamoto, D. Nishijima, Y. Ueda, et al., Nucl. Fusion 49 (2009) 065035. 

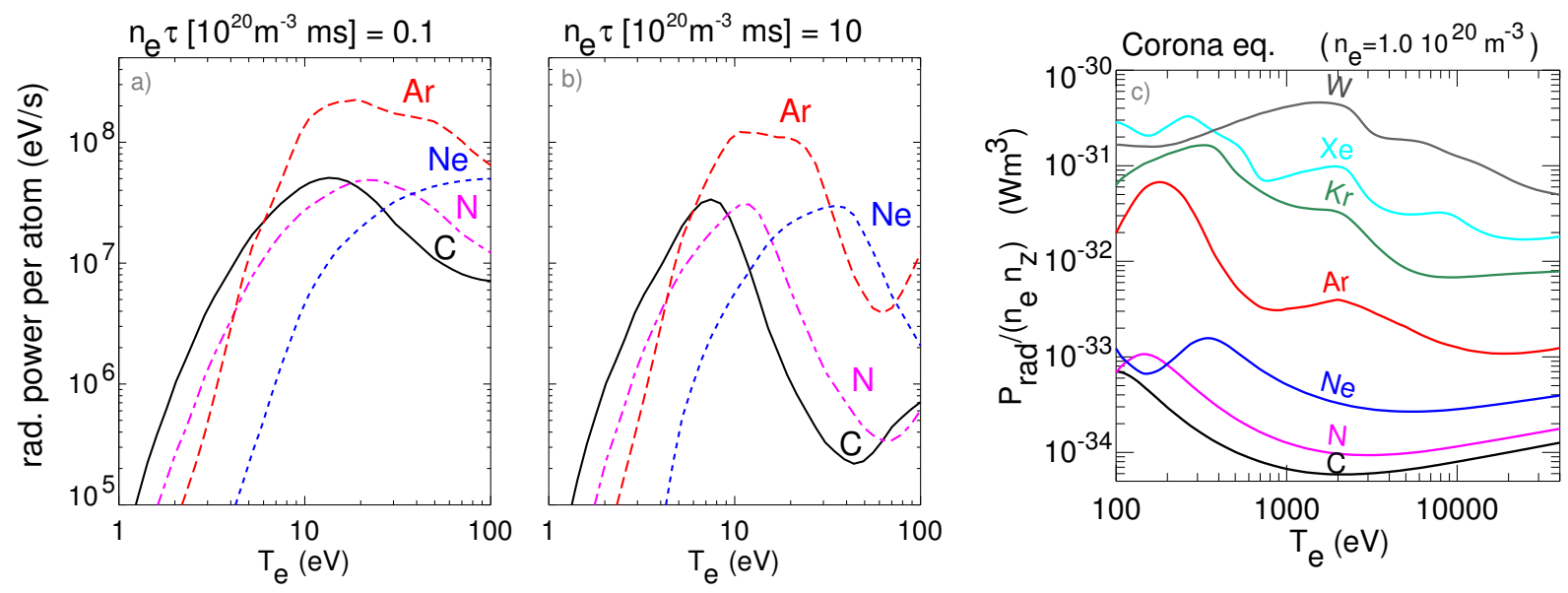

Fig. 1. Radiative loss factors for different seed impurities for divertor ( $a, b)$ and core conditions (c) from ADAS [16]. a) and b) are calculated following the radiative potential ansatz and depend in addition on the residence time $\tau$. The parameter $\mathrm{n}_{e} \tau$ is a measure for the ratio of the particle residence time $\tau$ and the collisional-radiative evolution time, which is proportional to $\mathrm{n}_{e}$. Given is the radiated power of an impurity atom started as neutral undergoing subsequent ionisation and excitation averaged over the time $\tau$. $\mathrm{n}_{e} \tau=0.1$ corresponds to typical AUG divertor conditions, $\mathrm{n}_{e} \tau=10$ to high density reactor divertor conditions. For large values of $\mathrm{n}_{e} \tau$, coronal equilibrium conditions are approached. Multiplication of the graphs shown with the assumed residence times $\tau=0.1$ and $10 \mathrm{~ms}$, respectively, gives the corresponding radiative potentials [17]. c) shows the coronal equilibrium radiative loss function which is valid in in good approximation for core conditions 


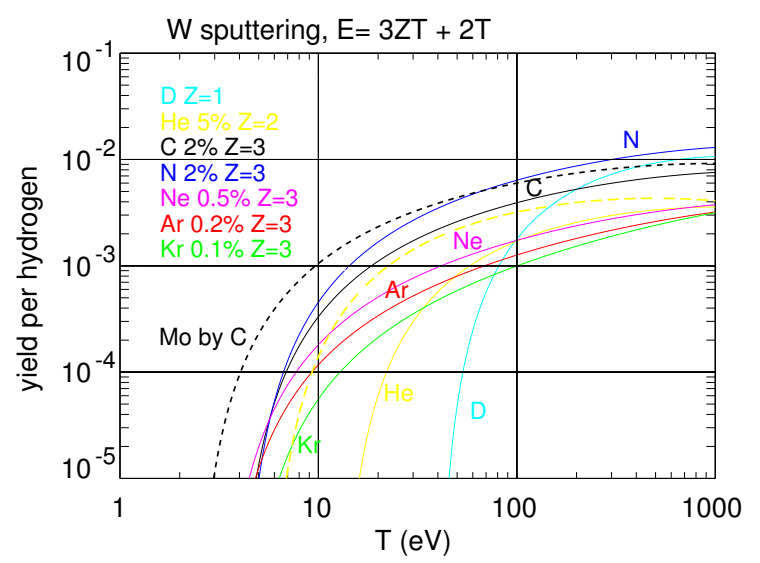

Fig. 2. Effective sputtering yields for tungsten by $\mathrm{D}, \mathrm{He}$ and different potential seed impurities, the molybdenum sputtering yield by $\mathrm{He}$ and $\mathrm{C}$ is indicated as dotted line for comparison. Yields have been multiplied with typical impurity concentrations expected for radiative high performance plasmas as indicated. These effective yields per hydrogen shown give the sputtered fluxes for the seed impurity concentrations assumed if multiplied with the (hydrogenic) ion wall flux. Division of the yields shown by the indicated concentration gives the absolute sputtering yield for the impurity species. Sputtering data are revised Bohdansky formula fits taken from [29], with the exception of the nitrogen data which have been obtained from TRIM calculations (see [30]). 

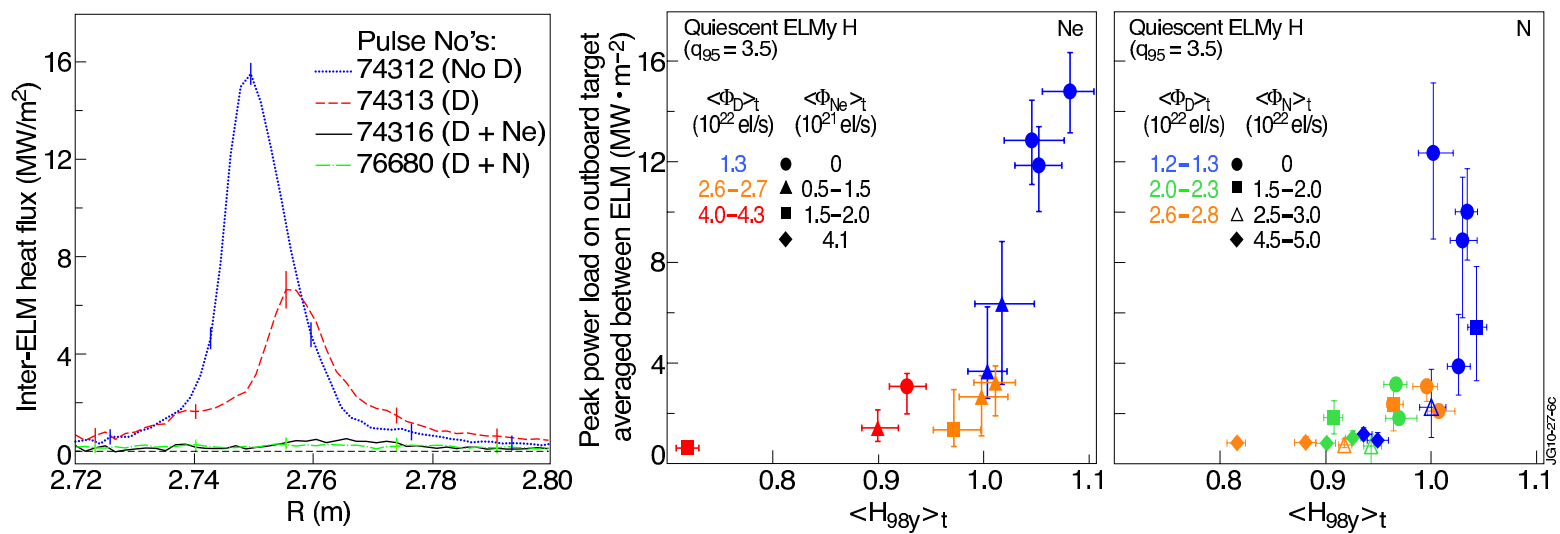

Fig. 3. Left: Inter-ELM heat flux profiles along the horizontal JET outer target. The highest heat flux is observed for unseeded conditions with very low D puff (labelled no D). The other cases have a D puff of $2.610^{22}$ at/s with no seeding and additional Ne and N seeding. $\mathrm{I}_{p}=2.5 \mathrm{MA}, \mathrm{B}_{t}=2.7 \mathrm{~T}, \mathrm{P}_{\text {heat }} \approx$ 16 MW. Mid: $\mathrm{H}_{98}$ vs. peak heat flux for Ne seeding. Right: same for $\mathrm{N}_{2}$ seeding. Data shown are from MHD-quiescent discharges, i.e. without strong MHD activity [33].
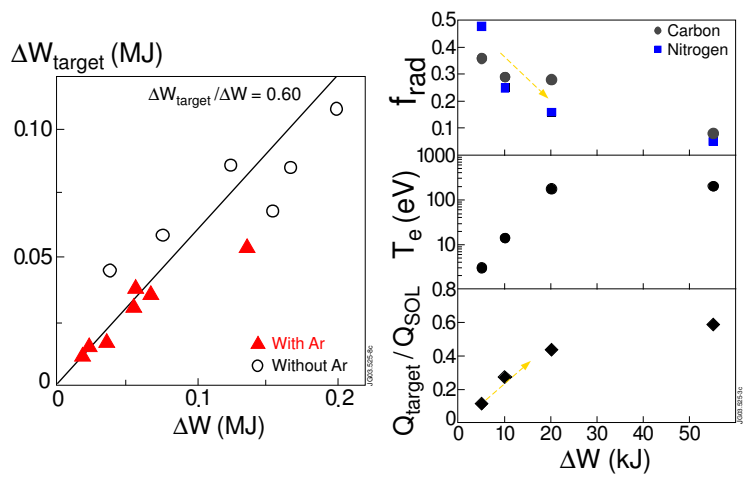

Fig. 4. Large ELMs cannot be buffered by radiation (from [40]). Left: ELM energy fraction arriving at the target with and without Ar seeding in JET. Right: Edge2d/NIMBUS modelling of ELM radiative buffering. Buffering is only effective for small ELMs below $20 \mathrm{~kJ}$. 


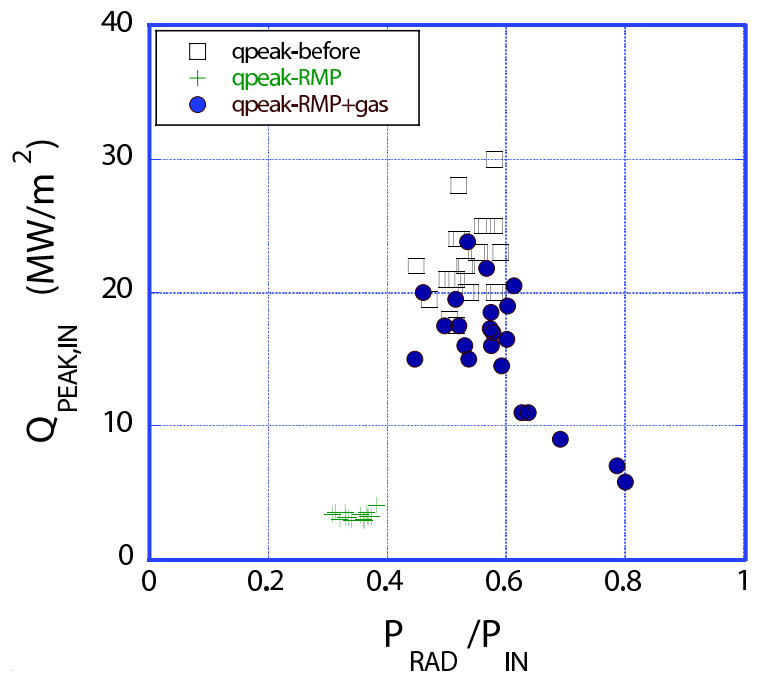

Fig. 5. Peak heat flux at the inner target during ELMs vs. radiative fraction for argon radiative divertor experiments in combination with RMP operation in DIII-D [43]. 

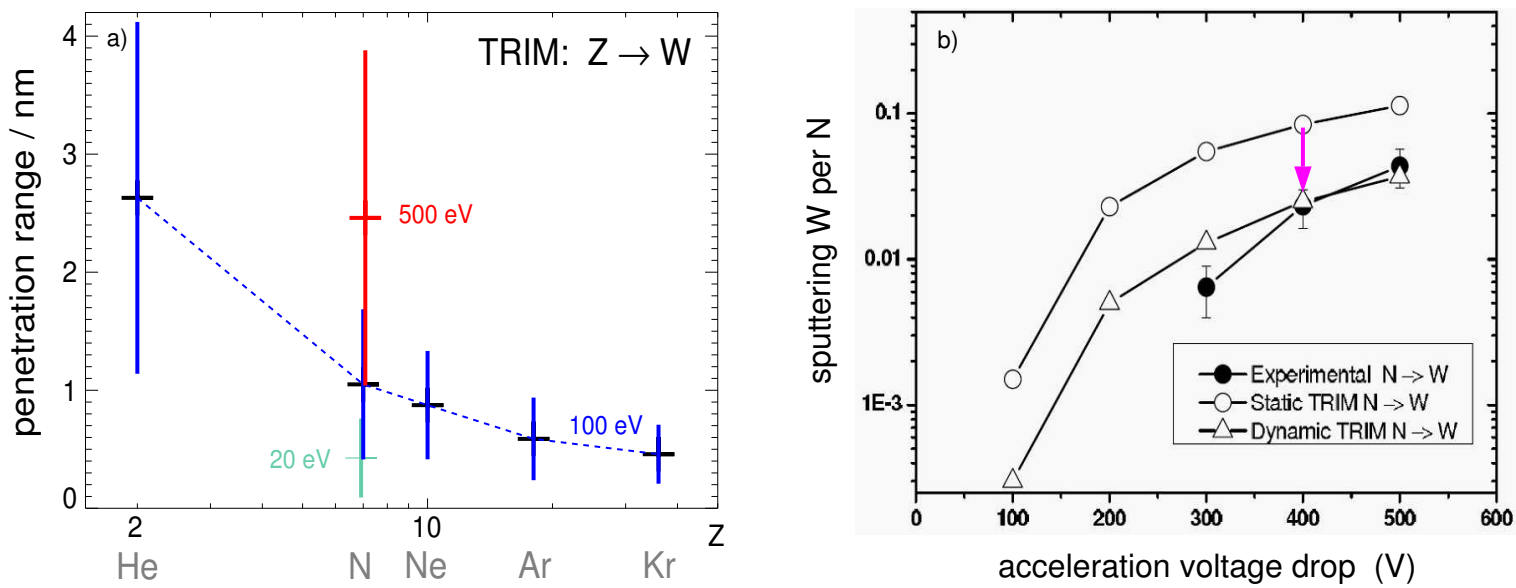

Fig. 6. a) Penetration depths of various seed impurity species in tungsten according to TRIM calculations. Mean projected ranges $\mathrm{R}_{p}$ are given for $100 \mathrm{eV}$ impact energy. For nitrogen, also the values for 20 and $500 \mathrm{eV}$ are plotted. The penetration depth increases approximately with the ion speed. The vertical bars show the standard deviation of the projected range. The separation of $\mathrm{W}$ atoms in the lattice is about $0.3 \mathrm{~nm}$. b) Measured and calculated sputter yields of $\mathrm{W}$ per impinging $\mathrm{N}$. The dynamic TRIM calculation takes the stored nitrogen into account [30]. 

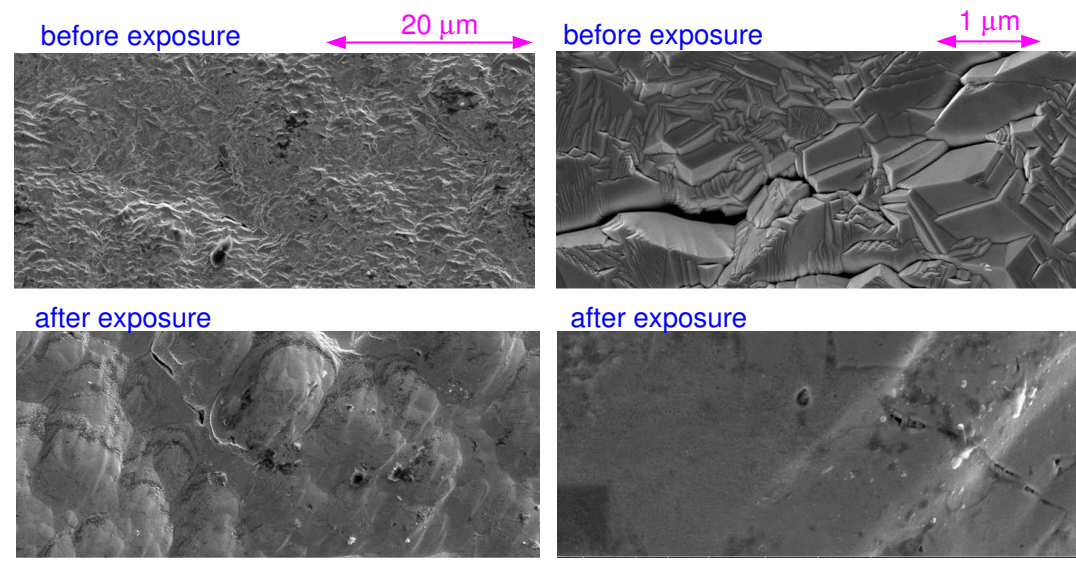

after exposure
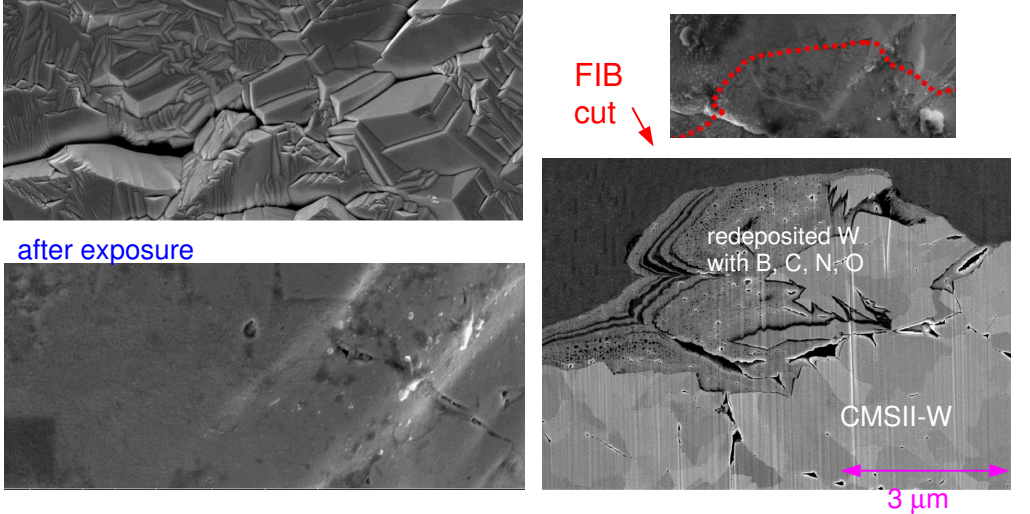

Fig. 7. Scanning electron micrograph of a tungsten outer divertor tile close to the strike point before and after plasma exposure. The W layer has a thickness of about $10 \mu \mathrm{m}$ and has been produced by CMSII (Combined magnetron sputtering and ion implantation [44]). Small scale roughness has been smoothed by plasma interaction. Large scale roughness has been partly eroded and produced redeposited $\mathrm{W}$ layers with $\mathrm{B}, \mathrm{C}, \mathrm{N}, \mathrm{O}$ embedded. These layers may contribute to the enhanced $\mathrm{N}$ storage under tokamak conditions. Measured net W erosion during a comparable previous campaign was $3 \mu \mathrm{m}$. 

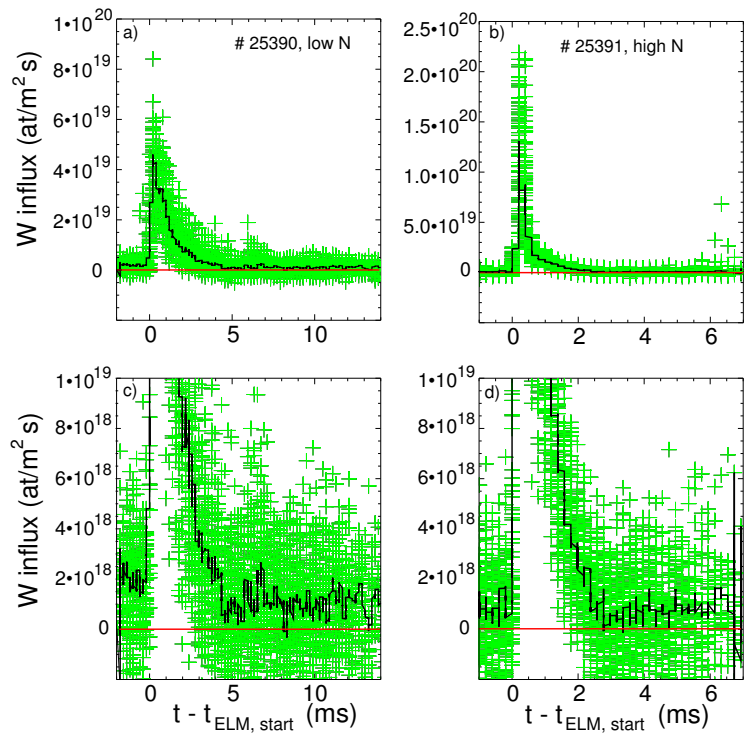

Fig. 8. WI flux from spectroscopy for 2 discharges with weak and strong nitrogen seeding. The lower curves have just expanded scales to make the inter-ELM fluxes visible. A constant value $S / X B=20$ was used to estimate particle fluxes from the measured photon fluxes of the $400.9 \mathrm{~nm}$ WI spectral line. No $\mathrm{T}_{e}$ dependence is employed, since these atomic data are currently under discussion [46]. The black line represents a coherent ELM average over $0.6 \mathrm{~s}$, crosses denote individual data points. 

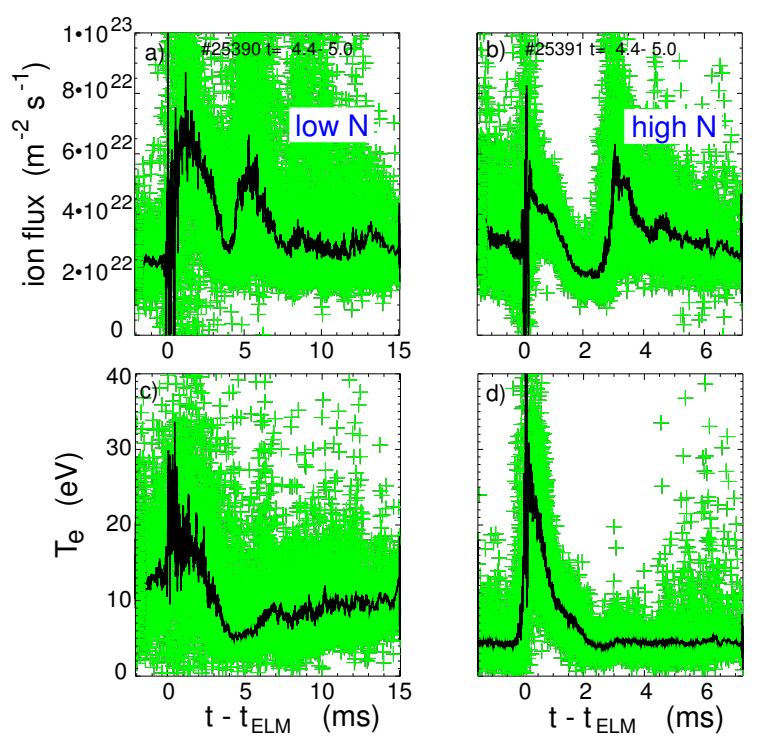

Fig. 9. Divertor parameters from a Langmuir probe over an ELM cycle close to the location of the WI flux shown in figure 8. a), b): ion saturation current for the discharges with low and high $\mathrm{N}$ puff rate. The second peak in the ion saturation current a few ms after the ELM is associated with a transient phase of higher recycling conditions [47], where $\mathrm{T}_{e}$ is reduced. c), $\mathrm{d}$ ): corresponding temporal evolution of the electron temperature.

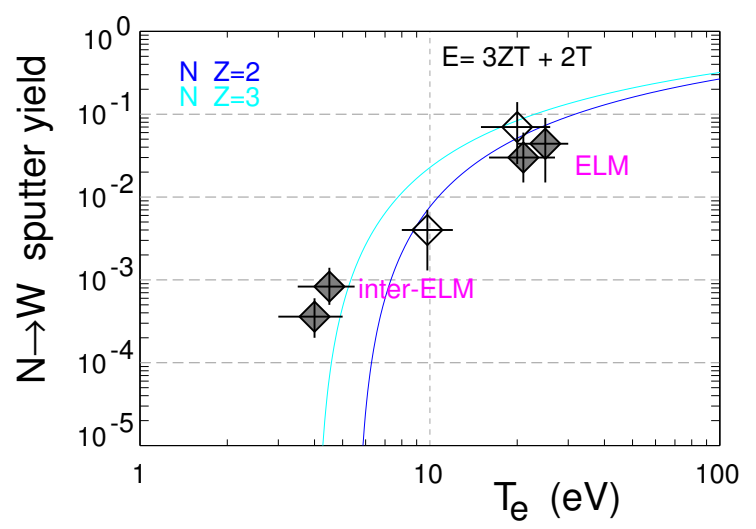

Fig. 10. Measured W sputtering yields for nitrogen seeded discharges versus an analytic fit of TRIM calculations for $\mathrm{N}^{2+}$ and $\mathrm{N}^{3+}$ ions. Tungsten influx and plasma parameters from figures 8 and 9. Solid symbols represent conditions close to $\mathrm{N}$-saturated tungsten divertor surface. The open symbols are not $\mathrm{N}$-saturated, but here intrinsic impurities contribute to the sputtered $\mathrm{W}$ flux. 